\title{
Robertsonian Translocation
}

National Cancer Institute

\section{Source}

National Cancer Institute. Robertsonian Translocation. NCI Thesaurus. Code C28447.

A translocation in which the centromeres of two chromosomes are fused, forming a

chromosome consisting of the long arms of two different chromosomes. -- 2003 